\title{
Treatment of tongue dysfunction: rehabilitation for prescribers' practice
}

\author{
H. Gil ${ }^{1}$, N. Fougeront ${ }^{2}$ \\ 1 Maxillofacial Physiotherapist, Lecturer at the dental faculty at Montrouge, University Paris \\ Descartes \\ 2 Dentist, Consultation of Orofacial Functional Disorders, Department of Oral Medicine/Dentistry, \\ Pitié-Salpétrière Charles-Foix Hospital Group, 94200 Ivry-sur-Seine, France
}

\begin{abstract}
Immature tongue function so-called "tongue-thrust or infantile and teeth apart swallow" and its rehabilitation involves multiple specialities in dentistry (pediatric dentistry, orthodontics, management of temporomandibular disorders, periodontics,...). Fifty years ago Mrs Fournier described a tongue rehabilitation technique. Given the difficulty to find a physiotherapist able to practice such a therapeutic, the aim of this article is to describe this therapeutic so that non-physiotherapists practitioners be able to initiate it and to teach it to their patients, at least for simple cases. It includes corrections of tongue immaturity, tongue resting position, deglutition and phonation. These changes might involve adaptive cortical neuroplasticity. Indeed these last years it has been shown in humans that standardised and calibrated tongue lift or protrusion exercises induce such plasticity in the tongue motor cortex.
\end{abstract}

\section{KEYWORDS}

Deglutition, phonation, tongue habits, rehabilitation, exercise therapy, neuronal plasticity

\section{INTRODUCTION}

The rehabilitation of tongue dysfunctions concerns many disciplines ${ }^{11}$ :

- pedodontics, orthodontics, and orthognathic surgery for the treatment of dysmorphosis

- ENT for the treatment of pathologies associated with dysmorphosis and obstructive sleep apnea syndrome
- the management of dysfunction of the masticatory apparatus,

- periodontology (aggravation of dental migrations by tongue dysfunctions) ${ }^{5}$.

The article "tongue dysfunction screening: assessment protocol for prescribers" 11 was written to highlight tongue dyspraxia dyspraxia and associated disorders. While

Address for correspondence:

This is an Open Access article distributed under the terms of the Creative Commons Attribution License (http://creativecommons.org/licenses/by/4.0), which permits unrestricted use, distribution, and reproduction in any medium, provided the original work is properly cited. 
this article deals with "diagnostic" aspects, the article below discusses the "treatment" aspect. In practice, a number of practitioners do not find referrals for their patients. To overcome this and to allow the patient to become aware of their tongue dyspraxia, tongue physiotherapy exercises have been adapted. These are indicated for cases of simple dyspraxia. It is also possible to indicate them as first intention, the time to address to a specialized physiotherapist.

\section{PRINCIPLES OF MAXILLOFACIAL REHABILITATION}

This specialty combines tongue rehabilitation (Fournier method) ${ }^{7}$ with Schultz's Autogenic Training ${ }^{15}$. The first aim is to correct the tongue according to Chateau's triptych principle 6,12 , i.e., at rest, swallowing, and phonation. At the same time, we will distance the periph- eral musculature, correct oral ventilation, and problems with general posture. Finally, the deep tensions will be treated by Schultz's relaxation technique. This is the most effective technique for eliminating bad habits and parafunctions. It is also called "therapeutic."

\section{REMINDER ON POSTURE AND LINGUAL PRAXIS}

Resting lingual posture is determined by a slight contact between the tip of the tongue, retroincisive papilla, and bunoid papillae on the palate. The edges of the tongue do not show any indentations. Physiological swallowing occurs on the palate with strong interdental contact and no muscular involvement of the labiojugal strap. In normal phonation, the $L, N, D$, and $T$ consonants are called "palatal" because the tip of the tongue touches the retroincisive area and bunoid papillae on the palate when these sounds are made.

\section{REHABILITATION}

\section{Aims}

The purpose of maxillofacial rehabilitation is to correct the poor tongue-posture and -praxis so that the teeth are found in a neutral dental corridor. There is then a balance between the centripetal force of the perioral musculature and the anteroposterior and transverse pressure of the tongue, guaranteeing

minimal and constant neuromuscular activity. We can talk about a real musculature tensioning procedure that helps to shape the dental arches. This corridor of influence is made up anteriorly by the labial and chin muscles and posteriorly by the masticatory muscles and laterally by the jugal muscles. Therefore, the tongue/lips, jaw-closing/jaw-opening muscles, and right and left muscle 
groups allow a harmonious growth of the bone architecture of the face and the alveolo-dental structures. At the same time, it is important to encourage nasal ventilation for wakefulness and sleep, to improve facial growth, harmonize spinal curvatures, improve sleep quality (apnea), and reduce any associated ENT pathologies.

\section{When to begin therapy?}

If the tongue dysfunction is isolated, the exercises recommended below may be prescribed by the odontologist and in many cases may correct this dyspraxia. On the other hand, if there are several associated disorders (ventilatory disorders, phonatory disorders, masticatory muscle pain, parafunctions, stress, etc.), it is advisable to contact a specialized physiotherapist.

\section{When to talk to a physiotherapist?}

When the patient has bad habits or tics, it is highly desirable to refer them to a physiotherapist trained in the Fournier and Schultz's relaxation techniques. If this is not the case, the physiotherapist can take charge of the correction of tongue dyspraxia and the patient will also have to undergo sophrology or hypnosis sessions.

\section{What to order?}

The wording of the order is assessment of the labial, jugal, lingual, swallowing, phonetic, ventilatory, postural, and temporomandibular joint functions, and rehabilitation if necessary. In general, the number of sessions is not to be specified.

\section{Number, duration, and frequency of sessions}

These treatment programs with the rehabilitator have a minimum of 20 sessions. It is essential that the odontologist be able to advise patients of the length and difficulty of this therapy. The first 10 sessions are devoted to learning new praxis and tongue posture. Additional sessions help verify that these techniques are working. Indeed, being able to perform the exercises voluntarily does not guarantee the success of this therapy. This is only obtained during the automation of new praxis and postures. As long as the corrections are not ingrained, it is necessary to continue the sessions. The duration of the session needs to be 30 min to obtain quality care and a privileged relationship with the patient. The patient is seen weekly at the beginning, then the sessions are spaced at 15 days, then monthly, until the corrections are automatically used.

\section{Follow-up}

An initial assessment is made at the first appointment to detect problems to be addressed. During treatment, intermediate assessments can be made on request and a final assessment will be sent to the practitioner to inform them of the progress, success, or failure of the treatment.

\section{Problems}

This treatment requires significant patient cooperation (treatment adherence and motivation). In addition, the practitioner will ensure long-term acquisition of results through 6-month and 1-year follow-up sessions. 


\section{AT WHAT AGE CAN WE BEGIN REHABILITATION?}

Maxillofacial rehabilitation differs according to patient's age and depends mainly on their motivation. Since a young child cannot practice exercises alone, automation cannot be achieved. In summary, children and adolescents are able to learn faster, but not necessarily well if they are not motivated, whereas adults learn better because they are more aware of the issues.

\section{APPROPRIATE METHODS FOR NON-PHYSIOTHERAPIST}

Whether it is a child or an adult, before starting any exercise, it is important to check that there is no tongue immaturity.

\section{How to detect lingual immaturity}

It is advisable to ask the patient to sweep their tongue along their palate from front to back, two to three times in a row and to describe the texture:

"Do you feel depressions, bumps; is it smooth or rough?" If the patient has difficulty performing this task or describing what they feel in their mouth, it is then advisable to make a gnosopraxic awakening so that they can acquire the control of their tongue and improve their proprioceptiveness.

\section{Exercises to overcome lingual immaturity}

Ask the patient to stick out their tongue, retract it, move it to the right, left, etc. It is necessary to vary the direction of movement and speed of execution. When the exercise is well done, ask the patient to "turn of the dials" by licking around their lips with the tip of the tongue 10 times in one direction and 10 times in the other. The exercise is properly performed when the tongue tip sweeps the vermilion of the lips, moving smoothly (Figs. 1 and 2). After that, the patient must perform the same gesture in front of a mirror, alternating one direction then the other. The difficulty of the exercise is increased by proposing to turn the lips toward the buccal surface 10 times in one direction, 10 times in the other, in one direction then in the other (Figs. 3 and 4).
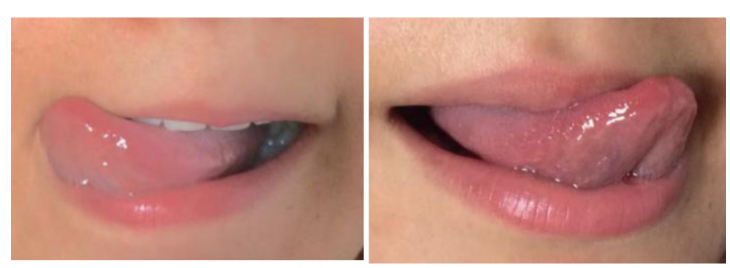

Figures 1 and 2

"Turn of the dials." The tip of the tongue licks around the lips.
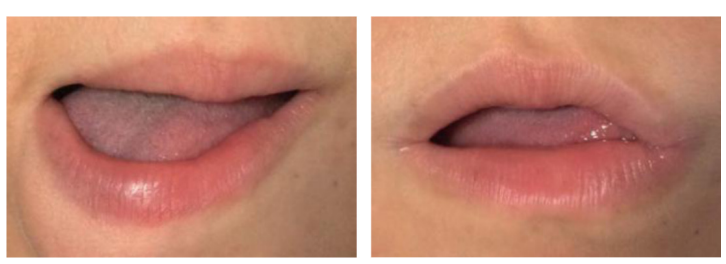

Figures 3 and 4

The tip of the tongue runs along the buccal edge of the teeth 10 times in one direction and 10 times in the other. 
When lingual immaturity is alleviated, we can teach them Chateau's tryptic ${ }^{6,12}$.

\section{Correction of lingual malposition at rest}

The patient learns to position the tip of their tongue on the retroincisive and bunoid papillae on the palate (Fig. 5). The contact is light and should not be forced. The tip is slightly flat on the papillae to prevent the tongue from folding back (Figs. 6-8). This position will be even more comfortable as the base of the tongue moves backward. This positioning should be done 10 times a day the first week, spread throughout

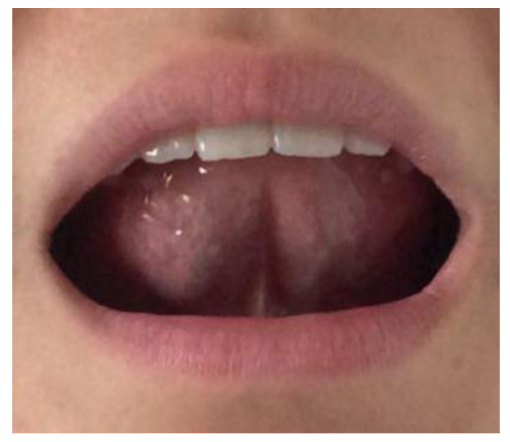

Figure 5

Position of the tongue tip on the retroincisive papilla. the day rather than 10 times in a row. In fact, it is an awareness, a correction of lingual malposition, and not an exercise that is to be repeated 10 times in a row "because it has to be done." With time, the patient is told to increase the number of corrections (ideally 20 times a day the second week, 30 times the third week, etc.) until the tongue feels at home on the palate. It can also be suggested to the patient to hold the tongue against the palate for as long as possible if they have difficulties and have to think about it often. To document the corrections, the patient is told to write down every day the number of times the tongue was repositioned. When the patient can do it 40 times a day, it means that the automation process has begun. In any case, when the patient feels any discomfort or pain, they must suspend the exercises.

\section{Correction of dysfunctional swallowing}

In addition to learning the correct resting lingual posture, the patient learns to swallow saliva with the tongue tip against the palate. Unlike at rest,
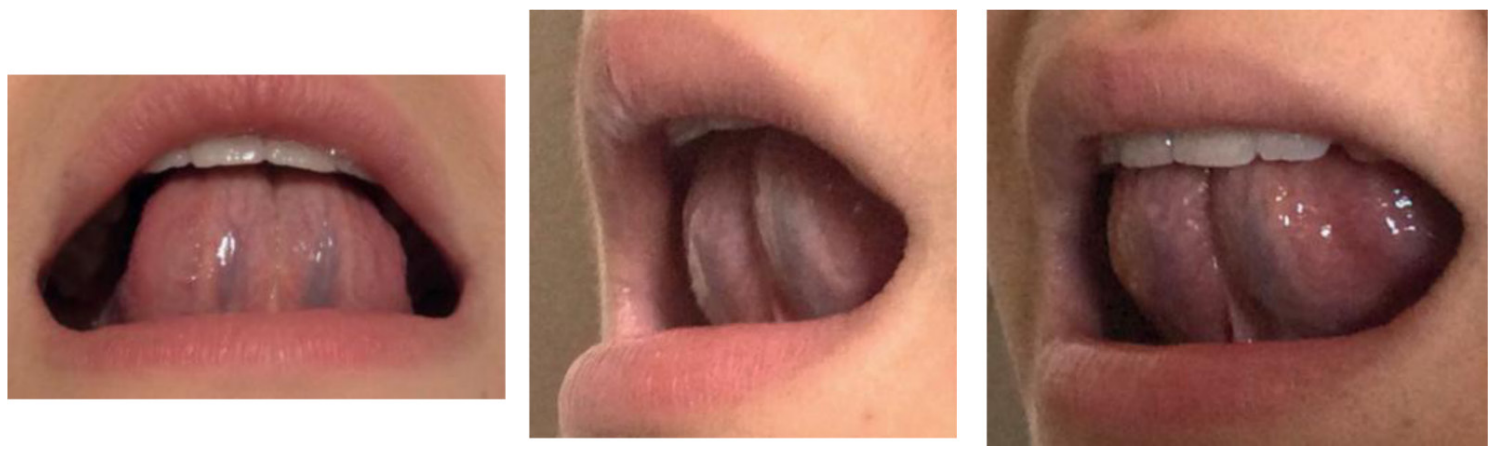

Figures 6-8

The tongue should not "stick outl" as shown above in Figures 1 and 2. 
support for the palate requires significant contact so that the tongue does not project onto the incisors. There is interdental contact, but no involvement of the cheek and lip strap. If, as a first step, the patient fails to use these peri-oral muscles (Fig. 9) (orbicularis of the mouth, modiolus, labiomental groove, or suction of the cheeks), they may try to swallow the saliva in non-occlusion. In case the gesture is still too difficult, "SLURP", i.e., strongly aspirating the saliva by emitting this sound and swallowing with an open mouth must be proposed. This will slide the saliva between the tongue and the palate, with labial non-occlusion keeping the perioral muscles at a distance. Since swallowing is a semiautomatic activity (approximately 2,000 times per day), the correction must be done in conjunction with the resting position of the tongue: each time the patient thinks of putting their tongue on the palate, they should try to swallow their saliva.

The correction of immature deglutition is completed by water sipping exercise (10 times in succession, once

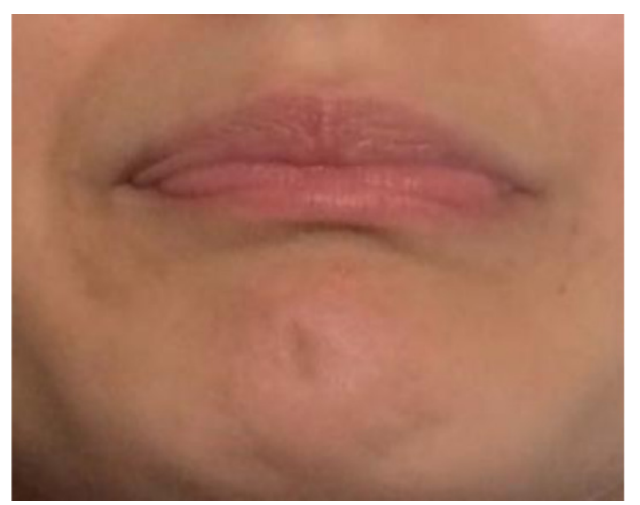

Figure 9

Swallowing with participation of the cheek and lip strap muscles.

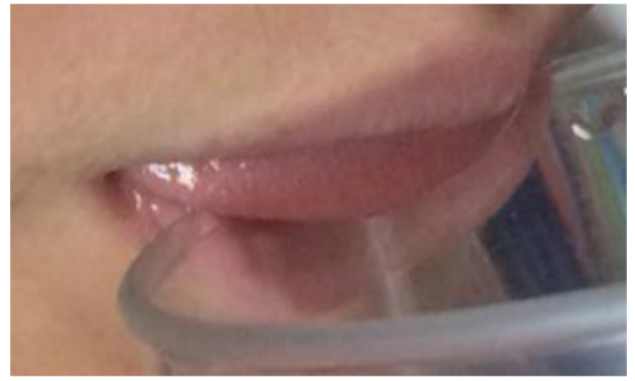

Figure 10

Before swallowing, protrusion of the tongue when approaching the glass of water.

a day). It involves taking a small sip of water from a glass and swallowing with the tongue on the palate, checking in the mirror that the perioral muscles do not contract. It is important to check that the patient is not propelling their tongue as they approach the glass of water (Fig. 10). In fact, this gesture reflects the "sucking actions." If this is the case, ask the patient to take a small sip of water while blocking the tongue at the palate from the start. When they have lost this lingual impulse upon approaching the glass, they can resume the first exercise. The difficulty is to gradually increase the amount of water in the glass and the speed of execution.

\section{Correction of phonation}

This consists of repeating aloud "LA, NA, DA, TA" (20 times in a row, once a day). The following week, the patient will double the number of phonemes"LALA, NANA, DADA, TATA" and finally "LALALALA, NANANANA, DADADADA, TATATATA." At the end of the rehabilitation, the patient must be careful not to touch their incisors while speaking and reading aloud. 


\section{The right attitude to maintain}

To complete Chateau's triptych, breathing must be nasal at rest, during exercise, day, and night. The patient should not feel any interdental contact at rest, especially in cases of bruxism. Spinal posture defects can also hinder

\section{THE EXPECTED RESULTS}

Rehabilitation is a success when the tongue is permanently on the palate. When reading or speaking, no immature deglutition occurs. The patient is able to swallow large sips of water without the tongue projecting onto the incisors and without the involvement

\section{LIMITS OF REHABILITATION}

Be it children or adults, problematic cases are often due to poorly invested or immature patients, with too busy schedules, states of tension that can affect the ingraining of corrections, ${ }^{12}$ unalleviated tongue immaturity, the the acquisition of good lingual posture. A tongue in the palate without correction of an anterior projection of the head does not facilitate the maintenance of the correct lingual posture in the palate. In this context, it is of interest to take care to prevent the accentuation of cervical, dorsal, and lumbar curvatures.

of the labiojugal strap muscles. When they speak or read with palatal $L, N, D$, and $T$ sounds, the tongue systematically involves the retroincisive and bunoid papillae. Oral ventilation, posture disorders, and parafunctions should then disappear.

presence in the palate of orthodontic appliances, and the repetitiveness of sessions. It is sometimes necessary to suspend the exercises and resume them later when the patient is more mature and more motivated.

\section{STANDARDIZED TONGUE EXERCISES:}

\section{A MODEL FOR STUDYING CORTICAL NEUROPLASTICITY}

Experimental study models might prefigure the mechanisms involved in functional rehabilitation in the clinic. Under experimental conditions in humans, standardized, calibrated, and low-intensity exercises tongue-protrusion or tongue-lift exercises induce neuroplastic changes in the brain, particularly the primary motor cortex (M1) the tongue primary motor cortex. Neuroplasticity or neuronal plasticity refers to functional (excitability), and long-lasting morphological modifications of neurons (gene expression 
modification, dendritic modifications, axon regrowth, and new synapses), changes of the intrinsic force and number of synapses, and changes in the neuronal trerritories. It can be either adaptive and beneficial, or maladptive and deleterious. This can happen throughout life (pain, motor learning, memorization, and development). In recent years, these phenomena have been extensively studied on the face, both in animals and humans. In humans, this plasticity is objectively determined by functional imaging ${ }^{2,4}$.

Tongue protrusion sessions of $1 \mathrm{~h}$ a day for a week ${ }^{16}$, as a single 1-h ses$\operatorname{sion}^{14,17}$, or even a session of just 15 $\mathrm{min}^{3}$, all help to improve the performance $1,3,14,16,17$, which leads to neuroplastic modifications of tongue $\mathrm{M}^{3,17}$. These phenomena of cortical neuroplasticity consist of an increased excitability of this cortical area $a^{3,14,16,17}$ and an increase in the size of this area ${ }^{16,17}$. Following these exercises, the tongue, in addition to $\mathrm{M} 1$ and other brain regions, is also the site of neuroplastic phenomena (supplementary motor area, putamen, thalamus, and cerebellum) ${ }^{1}$. It is remarkable that standardized tongue lift exercises not only induce neuroplastic neuroplastic changes of tongue $\mathrm{M} 1$ but also of masseter muscle M1, a muscle that has not been trained in this protocol ${ }^{13}$. Such an experimental protocol is similar to a mature tongue function (in the high position) where the activity of the masticatory muscles (trijeminal nerve - V) takes precedence over that of the facial muscles (facial nerve - VII). Conversely, during a lower lingual posture and immature tongue function, the activity of the platysma muscles predominates over that of the masticatory muscles. However, in the current state of clinical knowledge, we do not know the phenomena possibly implicated in lingual and maxillofacial rehabilitation in general as this is a new field of research ${ }^{8-10}$.

\section{CONCLUSION}

Although rehabilitation technique developed by Maryvonne Fournier is already 50 years old, it remains poorly understood. This may be due to skepticism from certain practitioners and/ or patients. In fact, its effectiveness essentially depends on the patient's cooperation. Its success also depends on the physiotherapist, who must be demanding in terms of controlling the results acquired while giving meaning to the methods taught (confidence and pleasure of learning for a better well-being). In the future, neuroscience will perhaps become clearer (phenomena of neuronal plasticity, modification of motor controls, and neuromuscular recruitment) and make it possible to understand why sometimes, despite the exercises, this method does not always work.

Conflict of interest: The authors declare that they have no conflict of interest 


\section{BIBLIOGRAPHY}

1. Arima T, Yanagi Y, Niddam DM, Ohata N, Arendt-Nielsen L, Minagi S, et al. Cortico motor plasticity induced by tongue-task training in humans: a longitudinal fMRIstudy. Exp Brain Res 2011;212(2):199-212.

2. Avivi-Arber L, Martin R, Lee J-C, Sessle BJ. Face sensori motor cortex and its neuroplasticity related to orofacial sensori motor functions. 2011. Arch Oral Biol 2011;56(12):1440-65.

3. Boudreau S, Romaniello A, Wang K, Svensson P, Sessle BJ, Arendt-Nielsen L. The effects of intraoral pain on motor cortex neuroplasticity associated with short-term novel tongue-protrusion training in humans. Pain 2007;132(1-2):169-78.

4. Boudreau SA, Farina D, Falla D. The role of motor learning and neuroplasticity in designing rehabilitation approaches for musculo skeletal pain disorders. Man Ther 2010;15(5):410-4.

5. Brunsvold MA. Pathologic tooth migration. J Periodontol 2005;76(6):859-66.

6. Chateau M. Orthopédie dento-faciale, tome 2, Clinique. Paris : Édition CdP, 1993:25-26.

7. Chauvois A, Fournier M, Girardin F. Rééducation des fonctions dans la thérapeutique orthodontique. Paris : Édition SID, 1991:77-78.

8. Fougeront N, Fleiter B. Localised muscle pain and dysfunction: a review of the oretical and suppositional biological effects of jaw exercises. Int J Stomatol Occlusion Medicine 2010;3(3):150-8.

9. Fougeront N, Fleiter B. Temporo mandibular disorders and co-morbid neck pain: facts and hypotheses regarding pain-induced and rehabilitation-induced motor activity changes. Can J Physiol Pharmacol 2018. DOI 10.1139/cjpp-2018-0100 (in press).

10. Fougeront N, Garnier B, Fleiter B. Rééducation fonctionnelle des troubles musculosquelettiques de l'appareil manducateur: de ses principes biologiques à la clinique (4e partie). Med Buccale Chir Buccale 2015;21(2):91-100.

11. Gil H. Fougeront N. Tongue dysfunction screening: assessment protocol for prescribers. J Dentofacial Anom Orthod 2015;18:408.

12. Gil H. Troubles temporo-mandibulaires d'origine musculaire et orthodontie. Rev Orthop Dento Faciale 2018;52:207-17.

13. Komoda Y, Lida T, Kothari M, Komiyama O, BaadHansen L, Kawara M, et al. Repeated tongue lift movement induces neuroplasticity in cortico motor control of tongue and jaw muscles in humans. Brain Res. 2015;1627:70-9.

14. Kothari M, Svensson P, Jensen J, Kjærsgaard A, Jeonghee K, Nielsen JF, et al. Traininginduced cortical plasticity compared between three tongue-training paradigms. Neuroscience 2013;246:1-12.

15. Schultz JH. Le training autogène. Paris : PUF, 1974.

16. Svensson P, Romaniello A, Arendt-Nielsen L, Sessle BJ. 2003. Plasticity in cortico motor control of the human tongue musculature induced by tongue-task training. Exp Brain Res. 152(1):42-51.

17. Svensson P, Romaniello A, Wang K, Arendt-Nielsen L, Sessle BJ. 2006. One hour of tongue-task training is associated with plasticity in cortico motor control of the human tongue musculature. Exp Brain Res. 173(1):165-73. 